\title{
Salutogenic Approach to Oral Health Promotion: A New Paradigm in Dental Public Health
}

\author{
Ali Hussain Khan* \\ Study conducted in Leeds Beckett University, Leeds, UK \\ *(Working as a Dental surgeon at the Aga Khan Health Services, Pakistan and Visiting Faculty at SZABIST).
}

Received: September 23, 2017; Accepted: October 10, 2017; Published: November 1, 2017

*Corresponding author: Ali Hussain Khan, Dental Surgeon at the Aga Khan Health Services, Pakistan. Telephone: +923369970912; Email: alihussain kh@gmail.com

\begin{abstract}
Oral Health Promotion (OHP) is one of the fundamental principles of the Ottawa Charter-an agreement signed for health promotion around the world. OHP has been studied though various public health theories, among which salutogenic theory has gained an important role in addressing the prevalence of oral health challenges and encouraging a multifaceted focus on preventing oral diseases that impact health and wellbeing. Salutogenic approach to oral health calls on Sense of Coherence (SOC) to identify possible ways to manage human behaviour, make right choices, prevent it from factors that harm, and create an environment favourable to good oral health and promotion oral health hygiene. To have a higher SOC means to have an ability to cope with stress, anxiety, poverty, disease. However, SOC is associated with the availability of resources- i.e. socio economic factors that determine our health based on the decisions, choices and actions individuals take. All these factors empower individuals towards manageability of their livelihood. To keep individual's SOC high, it is important to pay attention to the causes of their disease. The cause of oral disease is associated with the context of people's lives and changing the context of their lives could lead to higher SOC; hence, better oral health and wellbeing.
\end{abstract}

Keywords: Oral Health; Sense of Coherence; Public Health; Health Promotion;

\section{Introduction}

In the last two decades the salutogenic theory has gained ground in the field of oral health promotion. Although the two concepts, Salutogenesis and Oral Health Promotion, differ from one another, there are no conflicting points between the two concepts. The Sense of Coherence (SOC) framework is a practical theory for conducting research and practice on salutogenic approach to oral health promotion. On the contrary, the salutogenic way of thinking may strengthen the core principles of Health Promotion, since both approaches emphasise values such as equality, participation and empowerment by working towards creating possibilities for a good life and good oral health. In addition, population based approach is another health promoting principle with the focus on resources that enhance population ability promote and sustain good health and preventing negative health outcomes [1]. According to Eriksson \& Lindstrom, SOC is a health promoting resource linked with oral health outcomes. In order for people to make the right choices; increase control over their own lives and improve their social, mental and physical state, they need to be empowered. To be empowered means to have the ability to see oneself as an active participant in the society able to identify the use of internal and external resources, in order to meet and satisfy their needs as well as be able to choose and decide on their own [2]. The salutogenic approach could be therefore, a framework for empowerment contributing to the development of the SOC.

Oral diseases have tremendous impact on people's health and quality of life $[3,4]$. Oral health is a fundamental component of overall health and well-being [5-7]. Oral diseases are one of the current major public health problems due to high prevalence and direct social effects $[8,9]$. Towner states that in order to reduce oral diseases, most oral health promoters and dental practitioners use the conventional health education models based on clinical approach for explaining individual's life styles and treating patients [10]. In addition, oral health promoters adopted this approach for programme development, implementation and evaluation. These models; however, have serious limitations for showing sustained improvements in the field of oral health promotion [11]. Given that today is the period of evidence based oral health care, it is worth questioning the effectiveness and efficacy of current health educational methods. According to Labonte, many of them are unsuccessful in focusing on the environmental, political and social determinants of health [12].

Individuals' behaviours are enmeshed within the environment, socio-economic situations under which they live [13]. Individual's behaviours are widely influenced by these factors that subsequently impact their oral health outcomes [14]. To understand the variations in the oral health, it is important to not only focus on separate risk factors, but shift the focus 
towards people's life context - which is essential in oral health promotion [15]. Hence, in order to offer a good understanding of how different people perceive and assess their oral health, effective oral health promotion approach is needed for the prevention and promotion of oral health. Watt suggests a broader conceptualization of measuring oral health [16]. He suggests a new paradigm that has a multidimensional focus, most likely to be effective for enhancing populations' oral health, known as salutogenesis. Salutogenesis is considered as an upstream approach that challenges the dominant pathogenic paradigm, as it focuses on mechanism and pathways leading to good oral health [17].

Salutogenesis points us towards differentiating between factors promoting oral health and factors generating oral diseases. Hence, a person moving towards the health end of the continuum does not essentially mean that he/she is less exposed to risk factors related to oral diseases [18]. This means that in order for an individual to be healthy, the absence of risk factor (disease) is neither necessary nor sufficient. Yet, even if an individual is exposed to oral diseases, he/she can keep healthy due to his/her ability to have control over his/her life, which harmonizes well with the Ottawa Charter that focuses on the processes of enabling individuals to do so. For example, research shows that diabetes directly impacts oral health, but a patient with diabetes can maintain positive oral health by visiting the dentist regularly and teeth-brushing twice a day [19]. However, to visit the dentist and to afford teeth-brushing products every three months requires resources. This is a point where the salutogenic approach to oral health needs further investigation for finding ways for people to access resources. This is also emphasized by the Ottawa Charter that encourages oral health promoters to go beyond the health behaviour and health sector for generating quality of life and wellbeing. Eriksson and Lindström connect the Ottawa Charter with salutogenesis and emphasize the role of salutogenic principles and the concept of SOC in health promotion interventions for society, group, and individual health [17].

SOC offers a positive ways of looking at life along with the abilities to manage various stresses effectively [20]. The concept of SOC is very applicable in oral health promotion, because it is directed towards prevention rather than cure [21]. According to Eriksson \& Lindstrom, SOC is a health promoting resource linked with oral health outcomes. SOC is a personal orientation that defines ways individuals respond to stresses in life and maintain oral health [17]. According to Bernabé et al, people who have strong SOC have fewer decayed teeth, low level gingivitis, good perception of oral health $[24,25]$. Therefore, it is possible that those with the stronger SOC look at disease and its symptoms as manageable [26].

Antonovsky mention that SOC is an important determinant of sustaining individual's position on the health-dis/ease continuum and move towards the direction of the health end [27]. Various resources determine the strength and weaknesses of the SOC [28]. Antonovsky defines them as Generalized Resistance Resources (GRR). This means that those with stronger SOC are people with resources, and have the capacity to utilize those resources in a healthy manner. People who have weaker SOC can be classified as the opposite. SOC can promote resource and develop positive state of health and oral health. SOC could be considered as a coping agent [29].

The evidence shows that there is an association between stress and oral diseases [30]. SOC is believed to serve as a protective mechanism against perceived stress [31, 32]. Higher SOC shows that individuals have better coping ability and find appropriate solutions at times of problems and maintain good health and oral health [33]. Lazarus has criticized the concept of the SOC and its relationship with health [34]. By evaluating the SOC as a justification of individuals' living perfect lives and having high SOC, he states that it is not achievable in the world full of diseases, stress and deaths.

Those who are unable to cope with stress and depression often end up finding alcohol or cigarettes as coping agents, both detrimental to health [35]. Consumption of alcohol combined with smoking can lead to thirty times higher chances of oral cancer [36]. As a result, they lose the capacity to take responsibility for their own health showing weak SOC and also low manageability component, and become prone to poor oral health. However, Antonovsky does not want us to focus on the cause of disease, rather, on the origin of health [27]. He notes that having high SOC is not necessarily an indication of a healthy lifestyle, and the choice that is made when drinking alcohol or smoking depends on social and cultural factors as opposed to people's understanding of life [37]. However, socio-cultural factors that influence the specific behavior can also be part of impacting the origin of a high SOC, thus enhancing the chances of non -alcoholic and nonsmoking behavior for an individual with high SOC [27]. Hence, in order to deal with issues that affect oral health [due to alcohol or cigarettes], one needs to go back to the context of the individual to measure the subjective wellbeing, yet context can be both, the cure and the cause of health outcomes.

SOC behaviour pathway is related to oral health since oral diseases carry a strong behavioural element [38]. Several studies found that oral health behaviors such as daily frequency of sugar intake, regular dental attendance and regular tooth brushing frequency were significantly linked with SOC [39-41]. Hence, individuals with strong SOC have good oral health related behaviours. However, Lindmark [38] notes that an individual's behaviors pattern is largely shaped by cultural traditions [42]. Yet, Antonovsky argues that SOC is applicable cross culturally because it does not measure specific mechanisms of coping but overall disposition [43].

For the last few decades, there have been significant improvements in the field of oral health in around the developed countries; yet, social inequality in oral health still remains [44]. Phelan suggests that the most efficient strategy of improving population oral health, and reducing inequalities in oral health, is to project upstream policy interventions that reach across sectors and build an environment (socio-economic, cultural and 
physical) that promotes healthy life and wellbeing [45]. This can be done through "making healthy choices easier', empowerment approach, and population based approach, where the content and the structure of all approaches are salutogenic rather than healthy policy only for the health services.

The foundation of oral health promotion activities, according to Sheiham and Watt should be based on theoretical models at individuals and organizational levels that initiate the reorientation of oral health activities to the salutogenic approach [46]. Various activities that are driven to develop salutogenic factors could be created in different settings where individuals work, study and live. Salutogenesis helps identifying resources, promote living and working environments that are favourable for oral health. It is the availability of these resources that impact choices to adopt healthy behaviour [47]. These, for example, may include maximizing access to free sugar drinks and food at a reasonable price; emotional support from peers, friends and family and minimizing stress [48]. The extension of those resources leads to the expansion of healthy choices. However, as a concept, salutogenesis is not easily transferable to disadvantaged social groups, since the opportunities to develop SOC will not be developed due to lack of resources to draw upon. Another argument around the SOC is noted by Da Silva, that an increase in resources does not essentially mean that the SOC can be reinforced, since people must be empowered in terms of dealing with factors that influence their oral health [49].

\section{Empowerment process}

Oral health promoters can enable people to make healthy choices; however, despite the supportive environments, making healthy choice could be challenging if individuals do not feel in control over the environment [50]. Being in control means that they have the ability to have mastery over their choices that influence their oral health. It also means that they have high comprehensibility, manageability and meaningfulness, the three components of the SOC; leading to the likelihood for being healthy through the life span; all three impact health, oral health and wellbeing.

Abrahamsson and Ejlertsson [33] note that there is dynamic relationship between the three components of the SOC. They define comprehensibility as the capability to understand life events ordered and structured and it could strengthen by supporting people to increase the awareness about their health (I KNOW). Manageability is the feeling of managing the situation, and it is influenced when people are provided with the support to find resources (I CAN). Meaningfulness used as a motivational factor in the SOC that takes into account the importance of people's participation (I WANT). These components are fundamentally important when working with oral health promotion [8]. Strong SOC signifies that the three components are eminent in an individual. However, in rare case an individual can have various levels for the three components. They also play significant role in individuals' decision making process and contribute to improving oral health and wellbeing, as well as empower them to understand their capacity to take responsibility for their own oral health [19].
People gaining control over their lives reflects the empowerment process. However, scholars do not go into depth in clarifying the association between empowerment and the SOC. Yet, Koelen and Lindström note that empowerment provides continuous supportive process, where individuals and groups are enabled to modify situations, resources, and skills-all could be seen as a tool for the development of the SOC [51]. Nutbeam defines empowerment in health promotion as the process through which individuals have control over their choices and actions that impact their health [50].

Antonovsky's salutogenic approach could be a theoretical framework for empowerment [28], since it helps learning process while promoting health over time. Oral Health Promoters (OHPs) facilitate the process of empowerment through health education-which should be based on people's participation in their life context. The education process is promoted when information is manageable, comprehensible, and meaningful. Hence, activities related to oral health education must be able to promote understanding, through facilitating people to understand unhealthy beliefs related to their oral health, and encourage changes in their lifestyle [49].

\section{Population based approach}

Traditionally, for the prevention of oral diseases, the high risk /bio-medical paradigm has been widely dominant. The WHO criticized the dominance of this paradigm as it has overshadowed the more important population approach [52]. Strumpfer argues that accepting the population approach does not mean rejecting the bio-medical paradigm, because this approach can also serve people who have chronic illness [53]. Further research is needed on pathogens since it still continues to hold theoretical and practical implications. However, policies that are targeted to improve people's oral health should perhaps consider population strategy for oral health prevention as part of the salutogenic approach. According to Rose, the population approach addresses the underlying causes of diseases throughout the whole population [54]. In addition, the feasibility of healthy choices that policy may also allow, easier for people to adopt, which is as well emphasized by the salutogenic theory [16]. Among these policies enhancing laws and legislation on encouraging schools to adopt frameworks based on Health- Promoting Schools Network.

\section{School Oral Health Policies}

Milsom et al. have assessed the impact of schools dental screening in the UK on dental visits and treatment received [55]. The screening program based on risk factor approach had negligible impact on oral health and fails to tackle oral health inequalities. One of the effective ways to tackle inequality and generate positive oral health is to adopt the framework of healthpromoting schools [56]. Salutogenesis can be fundamental to this framework where oral health is integrated into the school curriculum in a coherent manner; as a result, facilitating the learning process that may impact oral health and well-being of school staff, communities, and families [49]. According to WHO, schools that are health promotion based can enhance 
their capacity to generate a setting that is healthy for learning, living and working [57]. Schools oral health policies can address resources and oral health challenges [58]. These policies may include: encouraging environments that provides psychological support, supervising tooth-brushing on a daily basis and ban on sales of harmful food and beverages in school vicinity to promote oral hygiene and a good oral health. According to Malikaew, the evaluations of Health Promotion School programs have confirmed major improvements in the outcomes of oral hygiene in the UK [59]. For example, previously, in England, Dental Days were used to be observed in schools that had very good impact on people's oral health, but nowadays, they are not there anymore. Dental hygiene practice, therefore, embraces salutogenic approach.

While schools have been traditionally used as an appropriate setting for oral health intervention, there are other settings such as colleges, community centres, places of worship, prisons and many more that can integrate salutogenic approach in their policies and practices [2]. These salutogenic settings can contribute to the accessibility of GRR and eventually, increase the capacity of using them for improving oral health and well-being [60].

\section{Conclusion}

From what is found in the current public health literature, it becomes evident that in Oral Health Promotion, integrating a Salutogenic approach and Sense of Coherence into the content and structure of policies related to health and oral health is fundamental. This can be done though 'making healthier choices easier', i.e empowerment approach, and school based approachall impact oral health behaviour and oral health inequalities by improving the quality of people's lives and contributing to their health and wellbeing. However, these policies and strategies require strong collaboration and cooperation between community and higher authorities such as government, market industries [that produce sugar containing beverages, alcohol, and cigarettes] and civil society.

Given that the availability of resource is essential to salutogenesis, government's role is important for ensuring equal distribution of resources and provision of incentives for deprived sections of the population. Eriksson and Lindström note that at the macro-level, the main challenge is about developing salutogenic healthy policies- necessary for building and sustaining the resources that generate health - and constructing the coherence needed in order to build a health promoting society [2]. Salutogenesis can further explain the persistence of inequalities in oral health and ways to promote a more resourceful approach to tackle them. Literature suggest that government should shift its focus from policies that are based on traditional bio-medical approach to salutogenesis and it should take into account the relationship between general health and oral health in the development and implementation of all health programs, policies, and practice. For example, the salutogenic approach can encourages local communities' participation in the development of oral health programs (via schools, hospitals, health centres) since the theory invites oral health promoters and communities to jointly identify problems and solutions by focusing on positive capabilities. This participation may enforce empowerment for people to have better control over their life and achieve full health potential.

Therefore, the salutogenic approach could guide the emerging field of health promotion to re-gain its focus presented by the Ottawa Charter; to understand health in terms of resource, participative method, enabling empowerment, as well as creation of supportive environment and human capabilities.

\section{Recommendations}

Firstly, the WHO should take serious steps toward strengthening the idea of Oral Health Promotion within policy and practice. One of the strategic steps could be to add the two concepts, good oral health and salutogenesis, into the schools national curriculums and national models of health.

Secondly, oral health promoters must avoid negative health determinants and focus on promoting salutary factors, such as educating individuals through community-based programs, extending appointment time in practices, investing on effective peer education. Oral health promoters should understand and adopt positive oral health practices that are orientated towards coping strategies, using client-centered approach. These practices are well reflected in the Ottawa Charter.

Lastly, using salutogenesis as a theoretical model, further study is required in order to better understand the components of the SOC that are found to have a positive impact on oral health. Since the level of the SOC depends on the ability to utilize various resources in different situations, future studies could emphasize on identifying resources in order to contribute to the SOC and its relation to oral health.

\section{Reference}

1. Morgan A and Ziglio E. Revitalising the evidence base for public health: an assets model. Promot Educ. 2007;2:17-22.

2. Eriksson M and Lindström B. Antonovsky's sense of coherence scale and its relation with quality of life: a systematic review. J Epidemiol Community Health. 2007;61(11):938-944.

3. Lindström B and Eriksson M. Contextualizing salutogenesis and Antonovsky in public health development. Health Promot Int. 2006;21(3):238-244.

4. The World Oral Health Report 2003: continuous improvement of oral health in the 21st century-the approach of the WHO Global Oral Health Programme. World Health Organization.2003;Geneva.

5. Lakschevitz F, Aboodi G, Tenenbaum H and Glogauer M. Diabetes and periodontal diseases: interplay and links. Curr Diabetes Rev. 2011;7(6):433-439.

6. Friedewald VE, Kornman KS, Beck JD, Genco R, Goldfine A, Libby P, et al. The American Journal of Cardiology and Journal of Periodontology Editors' Consensus: Periodontitis and Atherosclerotic Cardiovascular Disease目. J Periodontol.2009;80(7):1021-1032. 
7. Azarpazhooh A and Leake JL. Systematic review of the association between respiratory diseases and oral health. J Periodontol. 2006;77(9):1465-1482.

8. Khamaiseh A and ALBashtawy M. Oral health knowledge, attitudes, and practices among secondary school students. British Journal of School Nursing.2013;8(4):194-199.

9. Søggard AJ. Theories and models of health behaviour. Oral health promotion. Oxford: Oxford Medical Publication.1996:25-57.

10.Towner E. The history of dental health education: a case study of Britain. Oral health promotion. Oxford: Oxford University Press.1993.

11.Sprod AJ, Anderson R and Treasure E T. Effective oral health promotion: literature review. Cardiff: Health Promotion Wales.1996:20

12. Labonté, R. Health promotion in the near future: remembrances of activism past. Health Education Journal.1999;58(4):365-377.

13.Watt RG. Strategies and approaches in oral disease prevention and health promotion. Bull World Health Organ. 2005;83(9):711-718.

14.Petersen PE. The World Oral Health Report 2003: continuous improvement of oral health in the 21st century-the approach of the WHO Global Oral Health Programme. Community Dent Oral Epidemiol. 2003;31:3-23.

15. Yevlahova D and Satur J. Models for individual oral health promotion and their effectiveness: a systematic review. Aust Dent J. 2009;54(3):190-197. doi: 10.1111/j.1834-7819.2009.01118.x.

16. Watt RG. From victim blaming to upstream action: tackling the social determinants of oral health inequalities. Community dent oral epidemiol. 2007;35(1), 1-11.

17.Eriksson M, Lindström B. A salutogenic interpretation of the Ottawa Charter. Health PromotInt. 2008;23(2):190-199.

18. Morrison V, and Bennett P. An introduction to health psychology Pearson Education.2009.

19. Moore P, Orchard T, Guggenheimer J, and Weyant R. J. Diabetes and oral health promotion: a survey of disease prevention behaviors. J Am Dent Assoc. 2000;131(9):1333-1341.

20. García-Moya I. and Morgan A. The utility of salutogenesis for guiding health promotion: the case for young people's well-being. Health Promot Int. 2017;32(4):723-733. Doi: 10.1093/heapro/daw008.

21.Länsimies H, Pietilä A.M, Hietasola-Husu S and Kangasniemi M. A systematic review of adolescents' sense of coherence and health Scand J Caring Sci. 2017 Feb 3. doi: 10.1111/scs.12402

22. Antonovsky A. The structure and properties of the sense of coherence scale. Soc. Sci. Med.1993;36(6):725-733.

23. Watt RG. Emerging theories into the social determinants of health: implications for oral health promotion. Community Dent Oral Epidemiol. 2002;30(4):241-247.

24.Bernabé E, Watt RG., Sheiham A, Suominen AL, Vehkalahti MM, Nordblad A, et al. Childhood socioeconomic position, adult sense of coherence and tooth retention. Community Dent Oral Epidemiol. 2012;40(1):46-52. Doi: 10.1111/j.1600-0528.2011.00633.x.
25. Ayo-Yusuf OA, Reddy PS and Van den Borne BW. Adolescents' sense of coherence and smoking as longitudinal predictors of self-reported gingivitis. Journal of clinical periodontology.2008;35(11):931-937

26. Lindmark U, Wagman P, Wahlin C and Rolander B. Workplace health in dental care - a salutogenic approach. Int J Dent Hyg. 2016. Doi: 10.1111/idh.12257.

27. Antonovsky A. Unraveling the mystery of health: how people manage stress and stay well. Calif: Jossey-Bass publishers, 1st edition, 1987.

28. Antonovsky A. The salutogenic model as a theory to guide health promotion. Health Promot. Int. 1996;11(1):11-18.

29. Eriksson M and Lindstrom B. Validity of Antonovsky's Sense of Coherence Scale: A Systematic Review. J Epidemiol Community Health. 2005;59(6):460-466.

30. Klages U, Weber AG and Wehrbein H. Approximal plaque and gingival sulcus bleeding in routine dental care patients: relations to life stress, somatization and depression. J Clin Periodontol. 2005;32(6):575-582.

31. Roy P, Tremblay G, Robertson S and Houle J. "Do it all by myself": A salutogenic approach of masculine health practice among farming men coping with stress. Am J Mens Health. 2017;11(5):1536-1546. doi: $10.1177 / 1557988315619677$.

32. Gambetta-Tessini K, Mariño R, Morgan M, Evans W and Anderson V. Stress and health-promoting attributes in Australian, New Zealand, and Chilean dental students. J Dent Educ. 2013;77(6):801-9.

33. Abrahamsson A and Ejlertsson G. A salutogenic perspective could be of practical relevance for the prevention of smoking amongst pregnant women. Midwifery. 2002;18(4):323-331.

34. Lazarus RS. Emotion and adaptation. Oxford University Press.1991.

35. Midanik LT, Soghikian K, Ransom LJ, and Polen MR. Alcohol problems and sense of coherence among older adults. Soc Sci Med. 1992;34(1):43-48.

36. Parkin DM. 3. Cancers attributable to consumption of alcohol in the UK in 2010. Br J Cancer. 2011;105(2): S14-S18. Doi: 10.1038/ bjc.2011.476

37. Lindström B, \& Eriksson M. Salutogenesis. J Epidemiol Community Health. 2005;59(6):440-442

38. Lindmark U, Stegmayr B, Nilsson B, Lindahl B, and Johansson I. Food selection associated with sense of coherence in adults. Nutr J.2005;4:9.

39. Freire MC, Sheiham A, Hardy R. Adolescents' sense of coherence, oral health status, and oral health-related behaviours. Community Dent Oral Epidemiol 2001;29:204-212.

40. Nilsson L. Health promotion as school development. Scandinavian journal of nutrition. 2004;48(2):70-76.

41. Dorri M, Sheiham A, Hardy R, and Watt R. The relationship between Sense of Coherence and tooth brushing behaviours in Iranian adolescents in Mashhad. J Clin Periodontol. 2010;37(1):46-52. Doi: 10.1111/j.1600-051X.2009.01497.x.

42. Hugoson A, Koch G, Helkimo AN and Lundin SA. Caries prevalence and distribution in individuals aged 3-20 years in Jönköping, Sweden, over a 30-year period (1973-2003). Int J Paediatr Dent. 2008;18(1):18-26. 
43. Antonovsky A. The structure and properties of the sense of coherence scale. Soc. Sci. Med.1993;36(6):725-733.

44. Watt RG. From victim blaming to upstream action: tackling the socia determinants of oral health inequalities. Community Dent Oral Epidemiol. 2007;35(1):1-11.

45. Phelan JC, Link BG and Tehranifar P. Social conditions as fundamental causes of health inequalities theory, evidence, and policy implications. ] Health Soc Behav. 2010;51:28-40. Doi: 10.1177/0022146510383498.

46. Sheiham A and Watt RG. The common risk factor approach: a rational basis for promoting oral health. Community Dent Oral Epidemiol. 2000;28(6):399-406.

47. Ayo-Yusuf OA, Reddy PS and Van den Borne BW. Adolescents' sense of coherence and smoking as longitudinal predictors of self-reported gingivitis. Journal of clinical periodontology.2008;35(11):931-937.

48. Moysés ST, Moysés SJ, Watt RG and Sheiham A. Associations between health promoting schools' policies and indicators of oral health in Brazil. Health Promotion International.2003;18(3):209-218.

49. Silva AND, Mendonça MHMD and Vettore MV. A salutogenic approach to oral health promotion. Cad Saude Publica. 2008;24 4:s521-530.

50. Nutbeam D. Evaluating health promotion-progress, problems and solutions. Health promotion international.1998;13(1):27-44.

51. Koelen MA, and Lindström B. Making healthy choices easy choices: the role of empowerment. Eur J Clin Nutr. 2005;59(1):S10-15.
52. Beaglehole R, and Bonita R. Public health at the crossroads: which way forward? Lancet.1998;351(9102):590-592.

53. Strümpfer DJW. Salutogenesis: A new paradigm. South African Journal of Psychology.1990;20(4):265-276.

54. Rose G. The strategy of preventive medicine. Oxford: Oxford University Press.1992.

55. Milsom K, Blinkhorn A, Worthington H, Threlfall A, Buchanan K, Kearney-Mitchell, et al. The effectiveness of school dental screening: a cluster-randomized control trial. J Dent Res. 2006;85(10):924-928.

56. Jensen BB, Wolfgang D, and Goof B. The Application of Salutogenesis in Schools. The Handbook of Salutogenesis, Springer International Publishing, 2017:225-235.

57.WHO Global school health initiative. Health promoting schools. A healthy setting for living, learning and working. Geneva; World Health Organization:1998.

58. Kwan SY, Petersen PE, Pine CM, and Borutta A. Health-promoting schools: an opportunity for oral health promotion. Bulletin of the World Health organization.2005;83(9):677-685.

59. Malikaew P, Watt RG, and Sheiham A. Prevalence and factors associated with traumatic dental injuries (TDI) to anterior teeth of 11-13 year old Thai children. Community Dent Health. 2006;23(4):222-227.

60. Dooris M, Doherty S and Orme J. The application of salutogenesis in universities. The Handbook of Salutogenesis. Springer International Publishing. 2017:237-245. 